\title{
Millimeter per Hour
}

National Cancer Institute

\section{Source}

National Cancer Institute. Millimeter per Hour. NCI Thesaurus. Code C67419.

A unit of both speed (scalar) and velocity (vector), defined as the distance of one

millimeter travelled per unit time equal to one hour. 